\title{
Detailed Arterial Anatomy and Its Anastomoses of the Sphenoid Ridge and Olfactory Groove Meningiomas with Special Reference to the Recurrent Branches from the Ophthalmic Artery
}

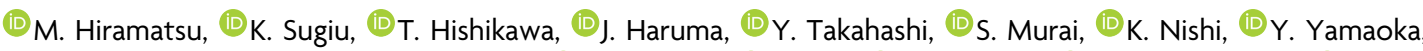

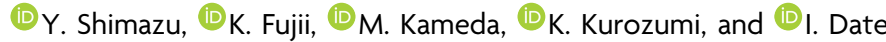

\begin{abstract}
BACKGROUND AND PURPOSE: Detailed arterial anatomy of the sphenoid ridge and olfactory groove meningiomas is complicated due to the fine angioarchitecture and anastomoses between each feeder. Herein, we present details of the arterial anatomy and the relationships of feeders in these lesions.
\end{abstract}

MATERIALS AND METHODS: This study included 20 patients admitted to our department between April 2015 and March 2020. Conditions of subjects consisted of 16 sphenoid ridge meningiomas and 4 olfactory groove meningiomas. We mainly analyzed arterial anatomy using 3D rotational angiography and slab MIP images of these lesions. We also analyzed the anastomoses of each feeder.

RESULTS: We found that 19 (95\%), 15 (75\%), and 15 (75\%) lesions had feeders from the ophthalmic, internal carotid, and external carotid arteries, respectively. As feeders from the ophthalmic artery, recurrent meningeal arteries were involved in 18 lesions (90\%). Fifteen lesions (75\%) had anastomoses between each feeder.

CONCLUSIONS: Most of the meningiomas in the sphenoid ridge and olfactory groove had feeders from the ophthalmic and internal carotid arteries. There were various anastomoses between each feeder. This is the first report to demonstrate the detailed arterial anatomy and frequency of recurrent branches from the ophthalmic artery and their anastomoses using detailed imaging techniques.

ABBREVIATIONS: ECA = external carotid artery; EtA = ethmoidal artery; ILT = inferolateral trunk; LSOF = lateral part of the superior orbital fissure; $\mathrm{MHT}=$ meningohypophyseal trunk; MMA = middle meningeal artery; MSOF = medial part of the superior orbital fissure; OC = optic canal; OphA = ophthalmic artery; RMA = recurrent meningeal artery; SOF = superior orbital fissure; TOB $=$ transosseous branch; WHO $=$ World Health Organization

$\mathbf{T}$ he dural arterial anatomy of the sphenoid ridge and anterior skull base is very complicated because there are many fine arteries from not only the external carotid artery (ECA) but also the ICA and ophthalmic artery (OphA). ${ }^{1}$ Moreover, there are various anastomoses between each artery. ${ }^{2-5}$ In the setting of hypervascular diseases related to the dura, such as meningioma or dural arteriovenous fistula in these regions, the angioarchitecture can be visualized

Received May 16, 2020; accepted after revision July 15.

From the Department of Neurological Surgery (M.H., K.S., T.H., J.H., Y.T., S.M., K.N., Y.Y., Y.S., K.F., M.K., K.K., I.D.), Okayama University Graduate School of Medicine, Dentistry and Pharmaceutical Sciences, Okayama, Japan; and Department of Neurosurgery (K.K.), Hamamatsu University School of Medicine, Hamamatsu, Shizuoka, Japan.

Please address correspondence to Masafumi Hiramatsu, MD, Department of Neurological Surgery, Okayama University Graduate School of Medicine, Dentistry and Pharmaceutical Sciences, 2-5-1 Shikata-cho, Kita-ku, Okayama 700-8558, Japan; e-mail: mhiramatsu@okayama-u.ac.jp

Indicates article with supplemental on-line table.

http://dx.doi.org/10.3174/ajnr.A6790 more clearly. In the present study, we investigated the details of the arterial anatomy of sphenoid ridge and olfactory groove meningiomas with special reference to the recurrent branches of the OphA and anastomoses between each feeder.

\section{MATERIALS AND METHODS}

All procedures performed in studies involving human participants were carried out in accordance with the ethical standards of Okayama University Graduate School of Medicine, Dentistry and Pharmaceutical Sciences's institutional research committee (1911-023). Written informed consent was obtained from all patients before DSA and treatment, but written informed consent for this study was not required because of the retrospective and noninvasive study design. Conditions of subjects consisted of 16 sphenoid ridge meningiomas and 4 olfactory groove meningiomas between April 2015 and March 2020. In this study period, we treated 29 patients with meningiomas in these regions, and we excluded 9 cases without 3D rotational DSA 


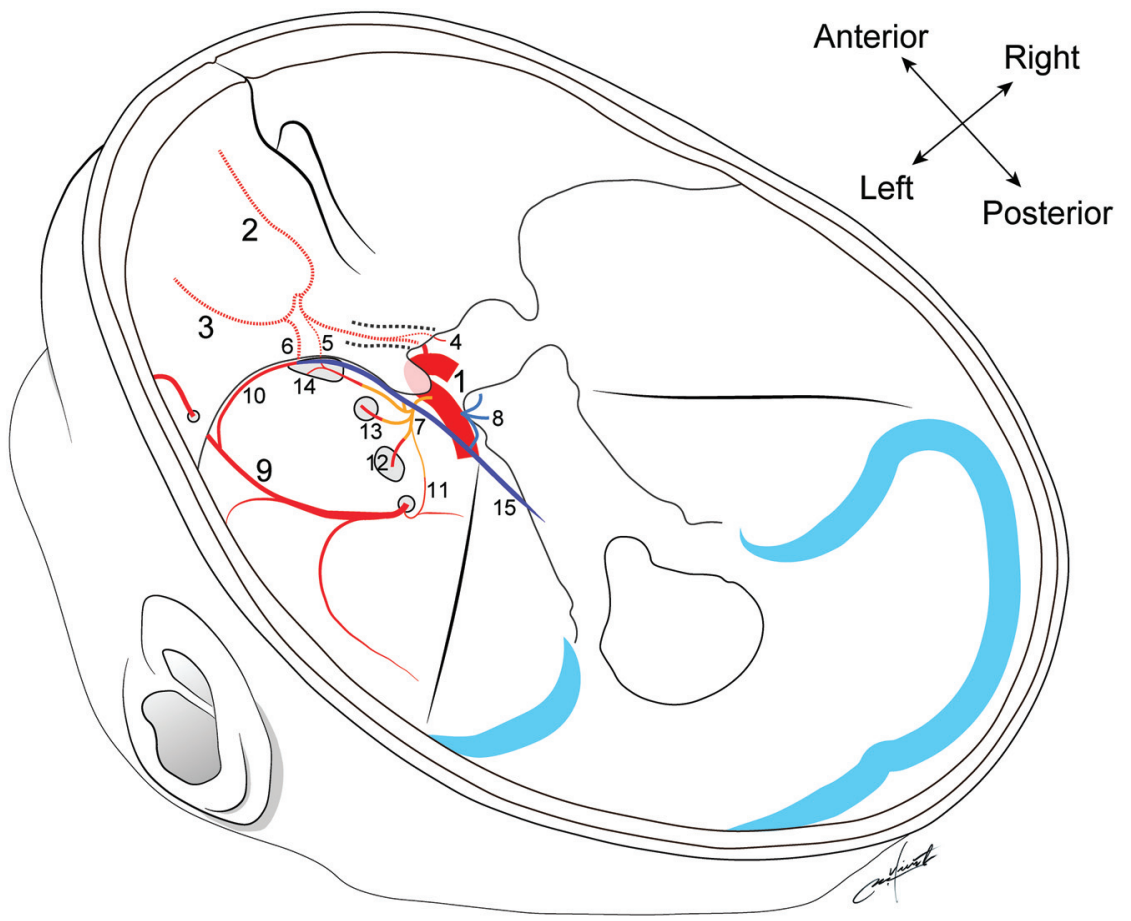

FIG 1. Schematic illustration of dural arterial anatomy and anastomoses among the ophthalmic, internal carotid, and external carotid arteries: 1 indicates the ICA; 2, OphA; 3, lacrimal artery; 4, RMA-OC; 5, RMA-MSOF; 6, RMA-LSOF; 7, ILT; 8, MHT; 9, MMA; 10, sphenoid branch of MMA; 11, cavernous branch of MMA; 12, accessory meningeal artery; 13, artery of the foramen rotundum; 14 , artery of the SOF; 15, marginal tentorial artery.

data because we could not analyze the details of the angioarchitecture in these cases.

We examined the meningioma location, tumor invasion, tumor size, histopathologic diagnosis according to World Health Organization (WHO) grade, and arterial anatomy of the feeders. Feeders were divided into branches of the OphA, ICA, and ECA (Fig 1). Branches of the OphA are the recurrent meningeal arteries (RMAs) described below, the ethmoidal artery (EtA), and the transosseous branch (TOB). Branches of the ICA are the inferolateral trunk (ILT) and the meningohypophyseal trunk (MHT). Branches of the ECA are the middle meningeal artery (MMA), accessory meningeal artery, deep temporal artery, artery of the foramen rotundum, artery of the superior orbital fissure(SOF), and sphenopalatine artery. We also analyzed the anastomoses of each feeder.

\section{Anatomic Terminology of the Branches of the Ophthalmic Artery}

We defined the RMAs as the posteriorly recurrent branches from the OphA or lacrimal artery passing through the SOF or optic canal (OC). We found 2 other types of feeders from the OphA. One of them was the TOB, which originates at the second or third portion of the OphA and courses superiorly, passing through the upper wall of the orbit to reach the dura of the anterior cranial base. Another one was the anterior or posterior EtA, which courses medially, passing through the ethmoidal foramina.

On the basis of the course, we divided feeders from the OphA into 5 types as follows: RMA passing through the OC (RMAOC), RMA passing through the lateral part of the SOF (RMA-
LSOF), RMA passing through the medial part of the SOF (RMA-MSOF), TOB, and EtA. We distinguished the RMA-LSOF and RMA-MSOF using the coronal section of slab MIP images from rotational angiography data. We present representative cases of the RMA-LSOF (Fig 2), RMA-OC (Figs 3 and 4), TOB (Fig 4), and EtA (Fig 4).

\section{Preoperative Examination}

Preoperative DSA was performed with the patient under local anesthesia. After obtaining 2D-DSA of the ECA and ICA, which are ipsilateral to the tumor, 3D-DSA with a 5-second protocol was performed on the branching feeders of the vessels. For 3D-DSA, a 4F diagnostic catheter was inserted into an ICA, and a $100 \%$ iodine contrast medium with a $300-\mathrm{mg} / \mathrm{mL}$ concentration was infused using an injector $(2.5 \mathrm{~mL} / \mathrm{s}$ flow rate, 1.5 - to 2.0-second delay, total 16$18 \mathrm{~mL}$ ). After obtaining volume-rendered images of 3D-DSA, slab MIP images were derived from rotational angiography data using a workstation to analyze details of the feeder anatomy. An Artis zee biplane (Siemens Healthcare) was used as a flat panel detector, and the syngo $\mathrm{X}$ Workplace (Siemens Healthcare) was used as a workstation.

\section{RESULTS}

The lesion characteristics and arterial anatomy of meningiomas are summarized in the Table, and details of each case are shown in On-line Table 1. In terms of lesion location, there were 4 sphenoid ridge lateral types, 9 sphenoid ridge medial types, 3 sphenoid ridge diffuse types spreading to the middle and anterior cranial base, and 4 olfactory groove lesions. There were 3 lesions with intraorbital invasion and 1 lesion with invasion to the temporal bone and muscle. The mean maximum diameter was $56 \mathrm{~mm}$. In terms of histopathologic diagnosis, there were 19 WHO grade I meningiomas and 1 WHO grade II meningioma. The 2 most frequent diagnoses were meningothelial $(n=8)$ and transitional $(n=8)$ meningiomas.

We found that 19 (95\%), 15 (75\%), and 15 (75\%) lesions had feeders from the OphA, ICA, and ECA, respectively. As the feeders from the OphA, there were 7 RMA-OCs in 7 lesions, 14 RMA-LSOFs in 13 lesions, 2 RMA-MSOFs in 2 lesions, 4 TOBs in 4 lesions, and 6 EtAs in 6 lesions. Six of 7 RMA-OCs (86\%) originated from the first segment of the OphA. Eleven of 14 RMA-LSOFs (79\%) originated from the lacrimal artery. Two RMA-MSOFs originated from the lacrimal artery and the second portion of the OphA. All (4/4) TOBs originated from the second or third segment of the OphA. Four of 6 EtAs (67\%) originated from the third segment of the OphA. RMAs from the OphA were involved in 18 lesions (90\%). All (7/7) RMA-OCs 

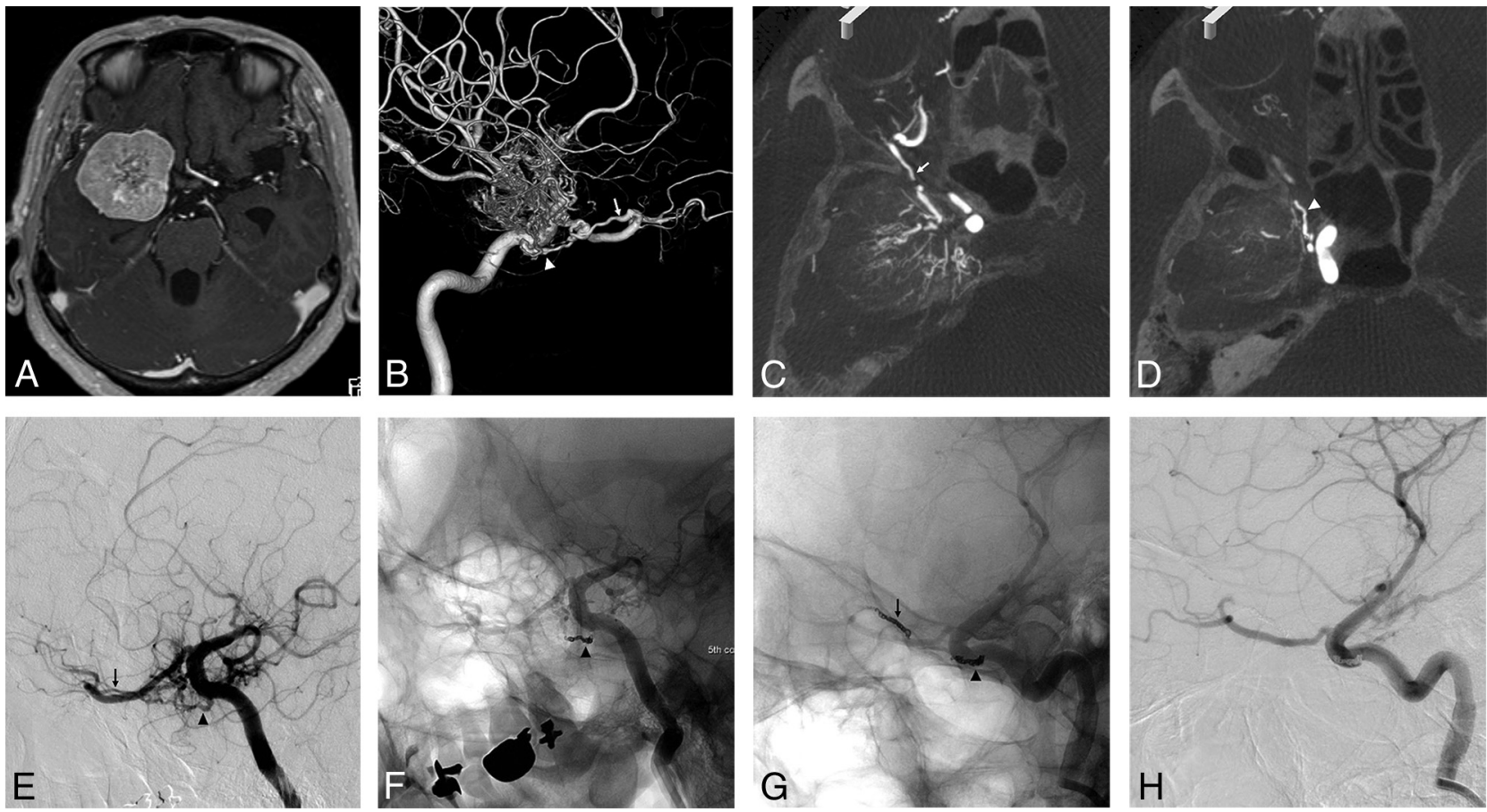

FIG 2. A, Axial view of the gadolinium-enhanced T1-weighted image shows a medial-type sphenoid ridge meningioma. The lateral view of the 3D-DSA $(B)$ and axial views of slab MIP images $(C$ and $D)$ of the right internal carotid angiogram show the feeder of the RMA passing through lateral part of the SOF (arrow) from the lacrimal artery and ILT (arrowhead). There is an anastomosis between the RMA-LSOF and the ILT. E, Oblique view of the internal carotid angiogram before embolization shows both feeders. Internal carotid angiogram after coil embolization of the ILT $(F)$ and RMA-LSOF ( $G$ and $H)$. The tumor stain is markedly diminished.
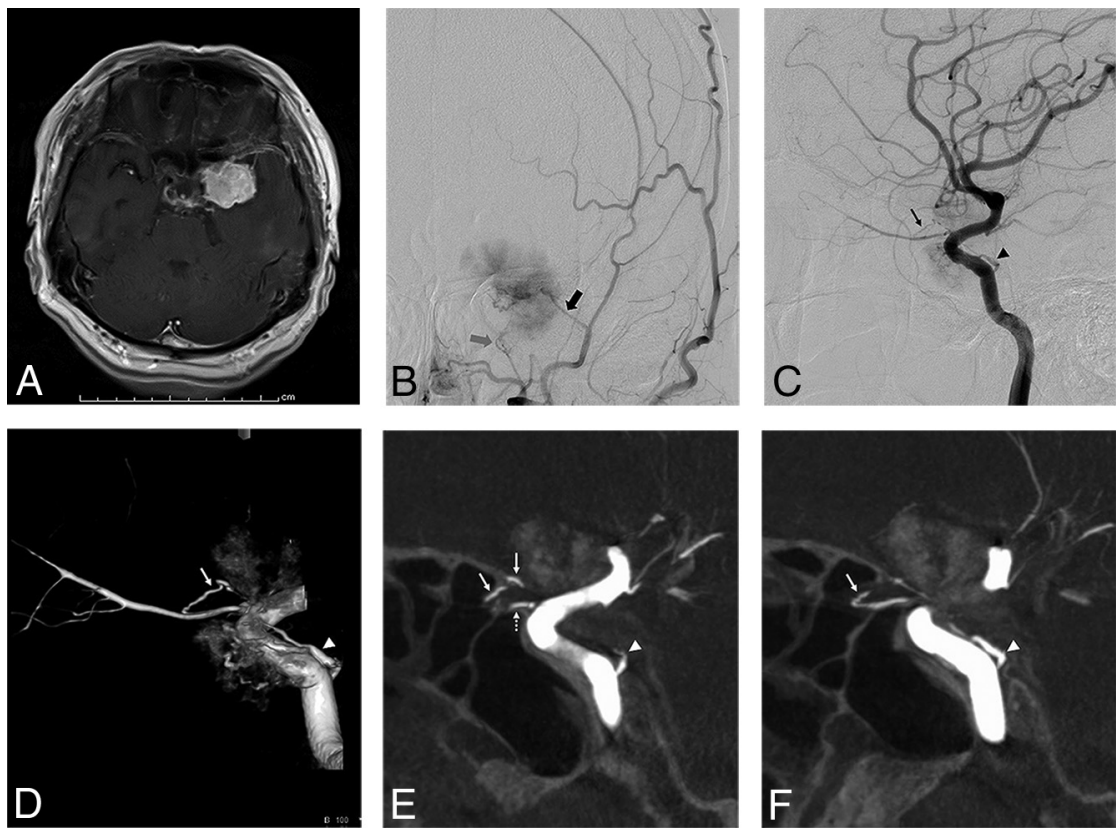

FIG 3. $A$, The axial view of the gadolinium-enhanced T1-weighted image shows a medial-type sphenoid ridge meningioma. $B$, Anterior-posterior view of the left external carotid angiogram shows the feeder from the accessory meningeal artery (large gray arrow) and sphenoid branch (large black arrow) of the MMA. The lateral views of the 2D-DSA (C) and 3D-DSA (D) and sagittal views of slab MIP images ( $E$ and $F$ ) of the left internal carotid angiogram show the feeder of the RMA passing through the OC (arrow) and MHT (arrowhead). The slab MIP images clearly show that the RMA-OC and OphA (dotted arrow) pass through the OC (E and F). appeared in medial-type sphenoid ridge meningiomas or olfactory groove meningiomas. RMA-LSOFs appeared in all types of sphenoid ridge meningiomas. All sphenoid ridge meningiomas with invasion to other locations (cases $1,2,8,15$ in the On-line Table) had multiple feeders from the OphA. One lesion (case 9 in the On-line Table) that had no feeder from the OphA was a psammomatous meningioma (WHO grade I). There were no other relationships between the arterial anatomy and invasion or histopathologic diagnosis.

As the feeders from the ICA, the ILT and MHT were involved in 13 and 9 lesions, respectively. As feeders from the ECA, the sphenoidal branch of the MMA, accessory meningeal artery, artery of the foramen rotundum, and artery of the SOF were involved in 12,7 , 6 , and 3 lesions, respectively. Other feeders from the ECA were the petrosal branch of the MMA, deep temporal artery, and sphenopalatine artery. There were 4 lesions with olfactory groove meningiomas. Most of the feeders of 

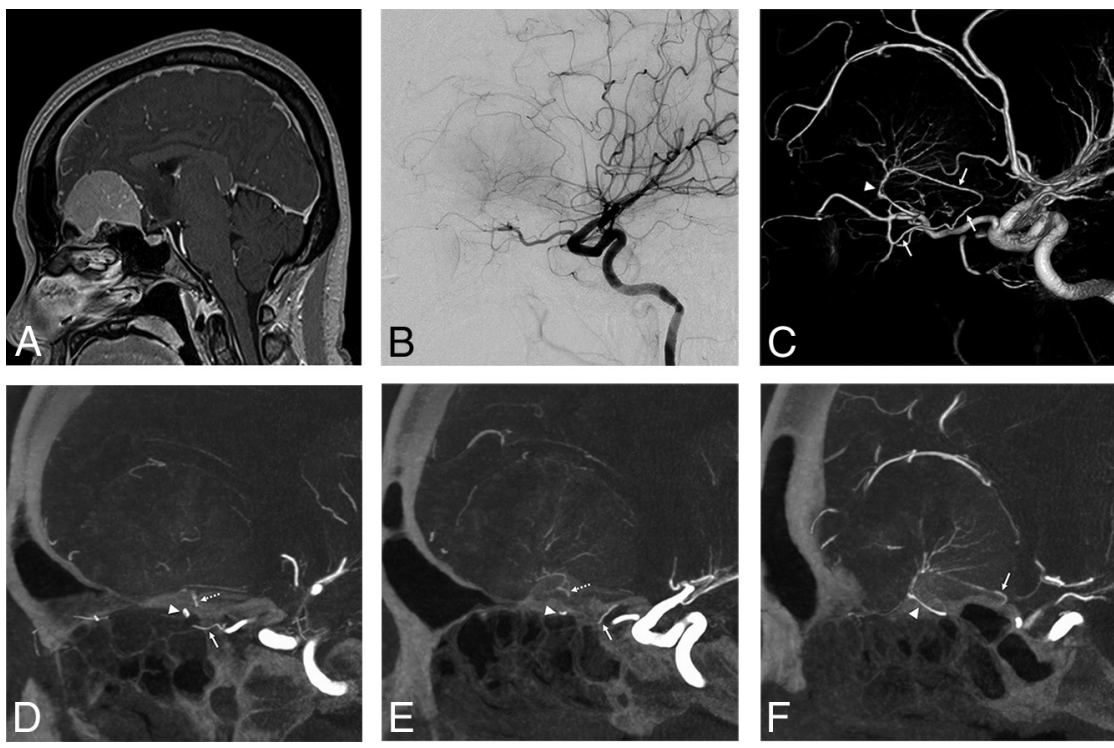

FIG 4. $A$, Sagittal view of the gadolinium-enhanced T1-weighted image shows an olfactory groove meningioma. The lateral views of the 2D-DSA (B) and 3D-DSA (C) and sagittal views of slab MIP images $(D-F)$ of the right internal carotid angiogram show the feeder from the RMA passing through the OC (arrow) from the third segment of the OphA, posterior EtA, (arrowhead), and TOB (dotted arrow). The slab MIP images show the RMA-OC, and the OphA passes through the $O C(E)$. Feeders from the RMA-OC and EtA configure an arcade shape on the blistering of the planum sphenoidale.

the olfactory groove meningioma were from the OphA, and there was no involvement of the ICA branches and MMA. As the feeders from the OphA, the EtAs, RMA-OCs, and TOBs were involved in 4,3 , and 1 lesion, respectively.

We found that 15 lesions (75\%) had anastomoses between each feeder. The most frequent anastomosis was between the OphA and the ICA (eg, RMA-LSOF and ILT). We also found anastomoses between feeders from the ipsilateral OphA (eg, RMA-OC and EtA). Five cases had anastomoses between the OphA or ICA and ECA. Four cases had multiple anastomoses, such as the RMA-ILT-MMA-artery of the SOF. In 3 cases of olfactory groove meningiomas, we found anastomosis between the RMA-OC and the EtA. The mean maximum diameter of the meningiomas that had anastomosis of feeders was $45 \pm 13 \mathrm{~mm}$ and that of meningiomas without anastomosis was $39 \pm 16 \mathrm{~mm}$.

\section{DISCUSSION}

Detailed arterial anatomy of the sphenoid ridge and the olfactory groove meningiomas is little understood due to the fine and complicated nature of arterial anatomy in the skull base. It is difficult to differentiate these subtle vessels using conventional 2D-DSA, 3DCTA, or MR imaging. We demonstrated that the latest imaging technique and slab MIP image of 3D rotational angiography can visualize this fine angioarchitecture. In the present study, we found that $95 \%, 75 \%$, and $75 \%$ of lesions have feeders from the OphA, ICA, and ECA, respectively. Moreover, we demonstrated that $75 \%$ of lesions have anastomoses between each feeder. Notably, this is the first report to demonstrate the detailed anatomy and frequency of recurrent branches from the OphA and their anastomoses using slab MIP images.

\section{Anatomy of the Dural Arteries}

Martins et $\mathrm{al}^{1}$ reported the detailed microsurgical anatomy of dural arteries as follows: The supply to the middle fossa and paracavernous dura derives laterally from the MMA, accessory meningeal artery, and ascending pharyngeal artery and medially from the intracavernous branches (ILT) of the ICA. They also described the contributions to these regions from the recurrent branches of the ophthalmic and lacrimal arteries. There is confusion about the definition of the recurrent ophthalmic arteries. Lasjaunias et $\mathrm{al}^{6}$ reported that both the superficial and deep recurrent ophthalmic arteries pass backward through the lateral and medial parts of the SOF, respectively. Meanwhile, Martins et $\mathrm{al}^{1}$ reported that the superficial recurrent ophthalmic artery, deep recurrent ophthalmic artery, and RMA pass through the optic canal, the medial part of the SOF, and the lateral part of the SOF, respectively. Therefore, descriptions of both authors were competing. In the present article, we describe the RMA as posteriorly recurrent branches from the OphA passing through the OC or SOF, including the superficial and deep recurrent ophthalmic arteries. Martins et $\mathrm{al}^{1}$ and $\mathrm{Hayreh}^{3}$ described the recurrent branch passing through the OC. However, to the best of our knowledge, no articles have shown this recurrent artery passing through the OC in imaging or cadaveric studies.

Yamaki et $\mathrm{al}^{7}$ reported details of feeding arteries in parasellar meningiomas, including 9 sphenoid ridge meningiomas. In this article, they analyzed 2 lesions (22\%) in the sphenoid ridge that were supplied from the superficial recurrent ophthalmic artery or recurrent meningeal artery, respectively. The current imaging technology and slab MIP images provide us with the ability to analyze detailed arterial anatomy. We can demonstrate that RMAs from the OphA were involved in most of the lesions (90\%) in the present study.

\section{Anastomosis of Each Feeder}

Numerous anastomoses between each artery have been reported. ${ }^{2-5}$ In the setting of hypervascular lesions related to the dura, such as meningiomas or dural arteriovenous fistulas, these feeding arteries can be enlarged and tend to connect. ${ }^{8}$ In the present study, there were various anastomoses in $75 \%$ of cases, and 4 cases had multiple anastomoses.

According to the embryologic concepts of the OphA, the connection between the OphA and the ILT is the remnant of the dorsal ophthalmic artery and the connection between the RMA and the MMA is the remnant of the supraorbital artery (branch of the 


\begin{tabular}{lc} 
Lesion characteristics and arterial anatomy of meningiomas \\
\hline \multicolumn{1}{c}{ Characteristics/Anatomy } \\
\hline Total & $n=20$ \\
Mean age (yr) & $56 \pm 14$ \\
Male sex (\%) & $9(45 \%)$ \\
Location (\%) & \\
Sphenoid ridge (lateral type) & $4(20 \%)$ \\
Sphenoid ridge (medial type) & $9(45 \%)$ \\
Sphenoid ridge (diffuse type) & $3(15 \%)$ \\
Olfactory groove & $4(20 \%)$ \\
Mean maximum diameter (mm) & $43 \pm 13$ \\
Histopathologic diagnosis (\%) & \\
Meningothelial meningioma & $8(40 \%)$ \\
Transitional meningioma & $8(40 \%)$ \\
Other WHO grade I meningioma & $3(15 \%)$ \\
WHO grade II meningioma & $1(5 \%)$ \\
Feeders from OphA (\%) & $19(95 \%)$ \\
RMA-OC & $7(35 \%)$ \\
RMA-LSOF & $13(65 \%)$ \\
RMA-MSOF & $2(10 \%)$ \\
EtA & $6(30 \%)$ \\
TOB & $4(20 \%)$ \\
Feeders from ICA (\%) & $15(75 \%)$ \\
ILT & $13(65 \%)$ \\
MHT & $9(45 \%)$ \\
Feeders from ECA (\%) & $15(75 \%)$ \\
MMA & $12(60 \%)$ \\
Accessory meningeal artery & $7(35 \%)$ \\
Artery of the foramen rotundum & $6(30 \%)$ \\
Artery of the SOF & $3(15 \%)$ \\
Anastomosis of each feeder (\%) & $15(75 \%)$ \\
OphA-OphA & $4(20 \%)$ \\
OphA-ICA & $5(25 \%)$ \\
OphA-ECA & $2(10 \%)$ \\
OphA-ICA-ECA & $2(10 \%)$ \\
ICA-ICA & $1(5 \%)$ \\
ICA-ECA & $1(5 \%)$ \\
\hline
\end{tabular}

stapedial artery). ${ }^{9-12}$ De La Torre and Netsky ${ }^{13}$ reported the persistent primitive maxillary artery in the human fetus. They reported that this artery arises from the cavernous portion of the ICA and anastomoses with the lacrimal artery and the anterior branch of the MMA. We can comprehend the complicated anastomoses among the OphA, ICA, and MMA using these findings. Therefore, it is certain that there are various anastomoses among branches of the OphA, ICA, and ECA.

\section{Clinical Implications}

Preoperative embolization for meningioma is performed as a standard treatment. Although its usefulness is widely accepted, the embolization of target vessels other than the ECA has been reported as a risk for procedural complications in a nationwide surveillance in Japan. ${ }^{14}$ Rosen et $\mathrm{al}^{15}$ reported their treatment results for preoperative embolization using microparticles to 167 cranial base meningiomas, and $9 \%$ of their patients experienced permanent neurologic deficits. They also showed that embolization of the MHT, MMA, and ascending pharyngeal artery involves the risk of a minor or permanent neurologic injury. Meanwhile, Waldron et $\mathrm{al}^{16}$ reported their treatment results for preoperative embolization using microparticles and coils to 119 cranial base meningiomas, with no complications. Some authors have reported the usefulness of embolization of the OphA branches. ${ }^{17,18}$ In limited cases, embolization may be performed for branches of the OphA, but it is associated with a risk of visual disturbance due to migration of the embolic material to the central retinal artery. ${ }^{17}$ Appropriate knowledge of anatomy, distal catheterization, and the gentle injection of embolic material without reflux are necessary for safe embolization of the OphA. ${ }^{19}$

In our opinion, the RMA-OC is inappropriate as the target vessel of preoperative embolization due to the difficulty in catheterization because of its small caliber and short distance from the origin of the OphA with an acute angle. The RMA-LSOF may have a lower risk because of its origins and anastomoses. The RMA-LSOF usually originates from a lacrimal artery and is anastomosed with the sphenoid branch of the MMA. However, if the RMA-LSOF has an anastomosis with the ILT, it involves a risk of the migration of particulate or liquid materials to the ICA. Thus, we recommend embolization of both of the RMA and the ILT using detachable coils in such a case.

Another precaution related to embolization is residual feeding from anastomotic vessels. If there is an anastomosis between each feeder, proximal occlusion of the feeder, which can be easily catheterized, can result in increased blood flow to the tumor from the residual feeder. One should embolize the common trunk of both feeders or the proximal parts of both feeders (Fig 2).

A detailed understanding of arterial microanatomy is also useful in direct surgery. A large sphenoid ridge meningioma requires skull base techniques, such as the transzygomatic approach. ${ }^{20,21}$ Using a skull base technique and extradural drilling of the sphenoid wing to the SOF, we can devascularize the feeder from the ECA and RMA-LSOF/MSOF. ${ }^{21}$ After these procedures, we open the dura mater, and we can debulk the intradural tumor, most of which is already devascularized, whereas, we may not be able to find the feeder from the RMA-OC until identification of the optic nerve. Moreover, coagulation to the RMA-OC involves the risk of optic nerve injury. If we identify all of the feeders using preoperative angiography, we can search for feeders on the basis of the anatomic landmarks of the bone and devascularize them in a safe and efficient manner.

\section{Limitations}

The current study has some limitations. First, the number of patients was small. Second, we did not confirm the presented feeding arteries with operative findings. In future studies, we should examine the accuracy of the presented results using operative findings or cadaveric research.

\section{CONCLUSIONS}

Sphenoid ridge and olfactory groove meningiomas have feeders from the OphA, ICA, and ECA in 95\%, 75\%, and 75\% of lesions, respectively. Furthermore, $75 \%$ of lesions have anastomoses between each feeder.

\section{ACKNOWLEDGMENTS}

The authors thank Dr Ryota Ishibashi (Department of Neurosurgery, Kurashiki Central Hospital, Kurashiki, Japan) for his suggestions on the writing of this article. 
Disclosures: Masafumi Hiramatsu-UNRELATED: Payment for Lectures Including Service on Speakers Bureaus: Siemens; Travel/Accommodations/Meeting Expenses Unrelated to Activities Listed: Siemens. Kenji Sugiu-UNRELATED: Payment for Lectures Including Service on Speakers Bureaus: Siemens; Travel/ Accommodations/Meeting Expenses Unrelated to Activities Listed: Siemens.

\section{REFERENCES}

1. Martins C, Yasuda A, Campero A, et al. Microsurgical anatomy of the dural arteries. Neurosurgery 2005;56(2 Suppl):211-51; discussion 211-51 CrossRef Medline

2. Geibprasert S, Pongpech S, Armstrong D, et al. Dangerous extracranial-intracranial anastomoses and supply to the cranial nerves: vessels the neurointerventionalist needs to know. AJNR Am J Neuroradiol 2009;30:1459-68 CrossRef Medline

3. Hayreh SS. Orbital vascular anatomy. Eye (Lond) 2006;20:1130-44 CrossRef Medline

4. Perrini P, Cardia A, Fraser K, et al. A microsurgical study of the anatomy and course of the ophthalmic artery and its possibly dangerous anastomoses. J Neurosurg 2007;106:142-50 CrossRef Medline

5. Toma N. Anatomy of the ophthalmic artery: embyrological consideration. Neurol Med Chir (Tokyo) 2016;56:585-91 CrossRef Medline

6. Lasjaunias P, Brismar J, Moret J, et al. Recurrent cavernous branches of the ophthalmic artery. Acta Radiology Diagn (Stockh) 1978;19:55360 CrossRef Medline

7. Yamaki T, Tanabe S, Sohma T, et al. Feeding arteries of parasellar meningiomas: angiographic study of medial sphenoid ridge and tuberculum sellae meningiomas. Neurol Med Chir (Tokyo) 1988;28:553-58 CrossRef Medline

8. Kiyosue H, Tanoue S, Hongo N, et al. Artery of the superior orbital fissure: an undescribed branch from the pterygopalatine segment of the maxillary artery to the orbital apex connecting with the anteromedial branch of the inferolateral trunk. AJNR Am J Neuroradiol 2015;36:1741-47 CrossRef Medline

9. Lasjaunias P, Berenstein A, terBrugge KG. Spinal artery. In: Lasjaunias P, Berenstein A, terBrugge KG. Surgical Neuroangiography, Volume 1; Clinical Vascular Anatomy and Variations. 2nd ed. Springer-Verlag; 2001:123
10. Lasjaunias P, Moret J, Mink J. The anatomy of the inferolateral trunk (ILT) of the internal carotid artery. Neuroradiology 1977;13:215-20 CrossRef Medline

11. Bonasia S, Bojanowski M, Robert T. Embryology and anatomical variations of the ophthalmic artery. Neuroradiology 2020;62:139-52 CrossRef Medline

12. Padget DH. The development of the cranial arteries in the human embryo. Contrib Embryol 1948;32:205-61

13. De La Torre E, Netsky MG. Study of persistent primitive maxillary artery in human fetus: Some homologies of cranial arteries in man and dog. Am J Anat 1960;106:185-95 CrossRef

14. Sugiu K, Hishikawa T, Murai S, et al. Treatment outcome of intracranial tumor embolization in Japan: Japanese Registry of NeuroEndovascular Therapy 3 (JR-NET3). Neurol Med Chir(Tokyo) 2019;59:41-47 CrossRef Medline

15. Rosen CL, Ammerman JM, Sekhar LN, et al. Outcome analysis of preoperative embolization in cranial base surgery. Acta Neurochir (Wien) 2002;144:1157-64 CrossRef Medline

16. Waldron JS, Sughrue ME, Hetts SW, et al. Embolization of skull base meningiomas and feeding vessels arising from the internal carotid circulation. Neurosurgery 2011;68:162-69 CrossRef Medline

17. Terada T, Kinoshita Y, Yokote H, et al. Preoperative embolization of meningiomas fed by ophthalmic branch arteries. Surg Neurol 1996;45:161-66 CrossRef Medline

18. Trivelatto F, Nakiri GS, Manisor M, et al. Preoperative Onyx embolization of meningiomas fed by the ophthalmic artery: a case series. AJNR Am J Neuroradiol 2011;32:1762-66 CrossRef Medline

19. Matsumaru Y, Alvarez H, Rodesch G, et al. Embolisation of branches of the ophthalmic artery. Interv Neuroradiol 1997;3:239-45 CrossRef Medline

20. Campero A, Villalonga JF, Elizalde RL, et al. Transzygomatic approach plus mini-peeling of middle fossa for devascularization of sphenoid wing meningiomas: technical note. Surg Neurol Int 2018;9:140 CrossRef Medline

21. González-Darder JM. Combined extradural and intradural pterional transzygomatic approach to large sphenoid wing meningiomas: operative technique and surgical results. J Neurol Surg B Skull Base 2019;80:244-51 CrossRef Medline 